\title{
Palmar main line polymorphisms in cataracts
}

\author{
T. PADMA \\ Ph.D.
}

J. S. Murty

Ph.D.

Department of Genetics, Osmania University, Hyderabad-500007, India

\begin{abstract}
Summary
The palm prints of patients with different types of cataract (total, nuclear, zonular, cortical and complicated) were analysed for main line terminations and compared with those of a random population. The cases were also grouped into congenital, juvenile and senile types for broader comparison.

The results showed significant variations for all the 4 main line terminations (D, C, B and A) in senile and cortical cataracts, while among other types of cataract, congenital cases differed significantly in $\mathbf{D}$ and $\mathbf{A}$ line, zonular in $\mathbf{C}$ and $\mathbf{A}$ line and nuclear in $B$ and $A$ line terminations compared with controls. A comparison of familial and isolated cases revealed significant differences for $D$ and $A$ line terminations only in cases of senile cataract. The main line terminations also indicated considerable sex-dependent heterogeneity among the diseased and control subjects.

The significance of the results obtained are discussed in terms of pleiotropic mechanisms leading to the formation of cataract.
\end{abstract}

\section{Introduction}

Terminations of palmar main lines-D, C, B and $A$ give an idea about the transverse and vertical alignment of ridges across the palm. These have until now been studied to delineate the differences between populations. Studies on the polymorphisms of main lines in diseases seem to be lacking except for a few reports such as that by Eswariah and Bali (1977) for diabetes. There are reports on the dermatoglyphic patterns in retinoblastoma (François et al., 1969; Vidal, Dameil and Funes, 1969) and cataract (Murty, 1973) which deal with characters other than the main line terminations.

The present authors studied the distribution of main line terminations in cases of cataract and compared them with those in a normal control group; they also studied the heterogeneity between various types of cataract. Cataracts have been selected for the study for 2 main reasons, namely, both lenticular and dermal tissues share a common embryonic origin and are derived from the ectoder mal layer during embryogenesis; and most of their lenticular structure is differentiated by the first trimester of fetal life while volar pads appearo between 6-8 weeks of gestation followed by theos ridge differentiation between the third and fourth\& fetal months. Therefore, an error in the genetico control of lens development is likely to be associatedwith a specific dermal ridge configuration.

\section{Material and methods}

Palm prints of 247 cataract patients reported at Sarojini Devi Eye Hospital and Institute of Ophth mology, Hyderabad, were analysed for variots. dermatoglyphic patterns. Of these, information ${ }^{\circ}$ palmar main line terminations was available for 206 cases (congenital, 67; juvenile, 30; senile, 109)음 which were analysed by the method of Cummins and Midlo (1943). Prints that lacked clarity were ${ }_{\Omega}^{\Phi}$ not included in the analysis. In cases of congenital $\Rightarrow$ cataract, prints were not taken of children below? the age of 12 months. The termination of each main? line was traced as illustrated in Fig. 1.

The cases were diagnosed for the specific type of cataract after slit lamp examination. Cases with 3 . suspected trauma, metabolic defects, diabetes, $\dot{\sigma}$ mental retardation, maternal infection during preg 3 . nancy and other associated ocular defects were $ᄋ$ eliminated from the study.

Detailed histories (including medical and genetico aspects) covering 2 to 5 generations were obtained, $>$ and revealed 40 cases with a positive family history, with the condition being present in first or second ${ }_{N}$ degree relatives. These $\mathbf{4 0}$ cases were classified as. ${ }^{\circ}$ genetic in origin and were grouped separately as $\tilde{O}$ 'familial' cases while the remainder without any N్ familial history were grouped as 'isolated' cases 0 (Padma et al., 1977). Of the 40 familial cases there were main line terminations in 36 cases, of which 14 were congenital; 7 , juvenile; and 15 , senile $\stackrel{?}{+}$ types. Information on parental phenotypes, incidence 0 of the condition in sibs, etc. in the familial cases are provided in Table 1. 


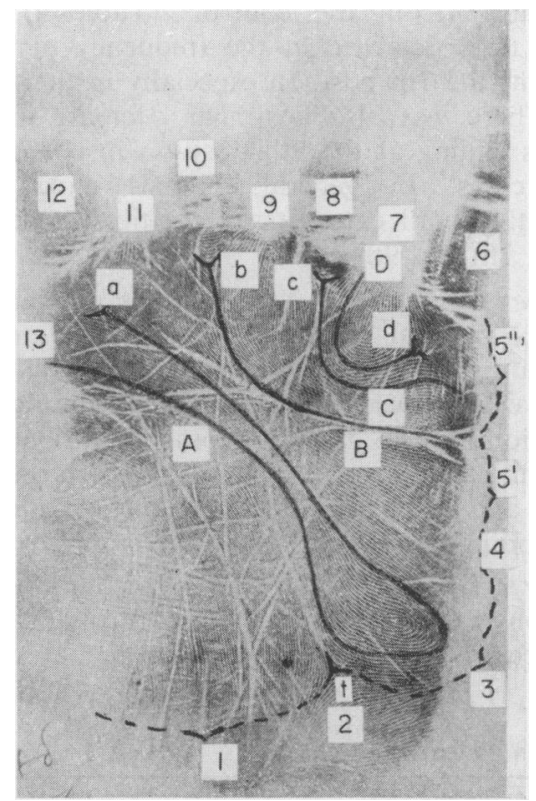

FIG. 1. Palm print showing the main lines (A, B, C, D) and their terminations.

A control group comprising 50 males (18 to 26 years) and 50 females (18 to 30 years) was also studied. The male controls were randomly selected from the inmates of the university hostels, while female controls constituted the normal mothers (selected randomly) of children suffering from diseases or defects other than ocular.
The sex distribution of the cases in the major groups of cataracts studied were as follows:

TABLE 2.

\begin{tabular}{lcccccc}
\hline $\begin{array}{l}\text { Type of } \\
\text { cataract }\end{array}$ & \multicolumn{3}{c}{ Familial } & \multicolumn{3}{c}{ Isolated } \\
\hline Congenital & 7 & 7 & 14 & 33 & 20 & 53 \\
Juvenile & 5 & 2 & 7 & 11 & 12 & 23 \\
Senile & 8 & 7 & 15 & 33 & 61 & 94 \\
Total & 20 & 16 & 36 & 77 & 93 & 170 \\
\hline
\end{tabular}

At the time of examination, the ages of congenital cataract cases ranged from a few days to 8 years. The diagnosis in children older than 3 years was based on (a) earlier medical history, (b) course of development of the cataract and (c) clinical picture as observed under slit lamp. Some of the cases were aphacic for one eye and were admitted to the hospital for surgery to the second eye. Cases which showed onset of cataract symptoms after 10 years but before 40 years of age were grouped as juvenile types, whereas cases which showed these after the age of 40 years were grouped as senile types.

The terminations of main lines in familial and in isolated cases were also compared. These details are given in Tables 3 to 6 for congenital, juvenile and senile cataracts only: the number of cases of 'other' cataracts studied was very small. While computing the $\chi^{2}$ values, points of terminations

TABLE 1. Family information of the patients with different types of cataracts

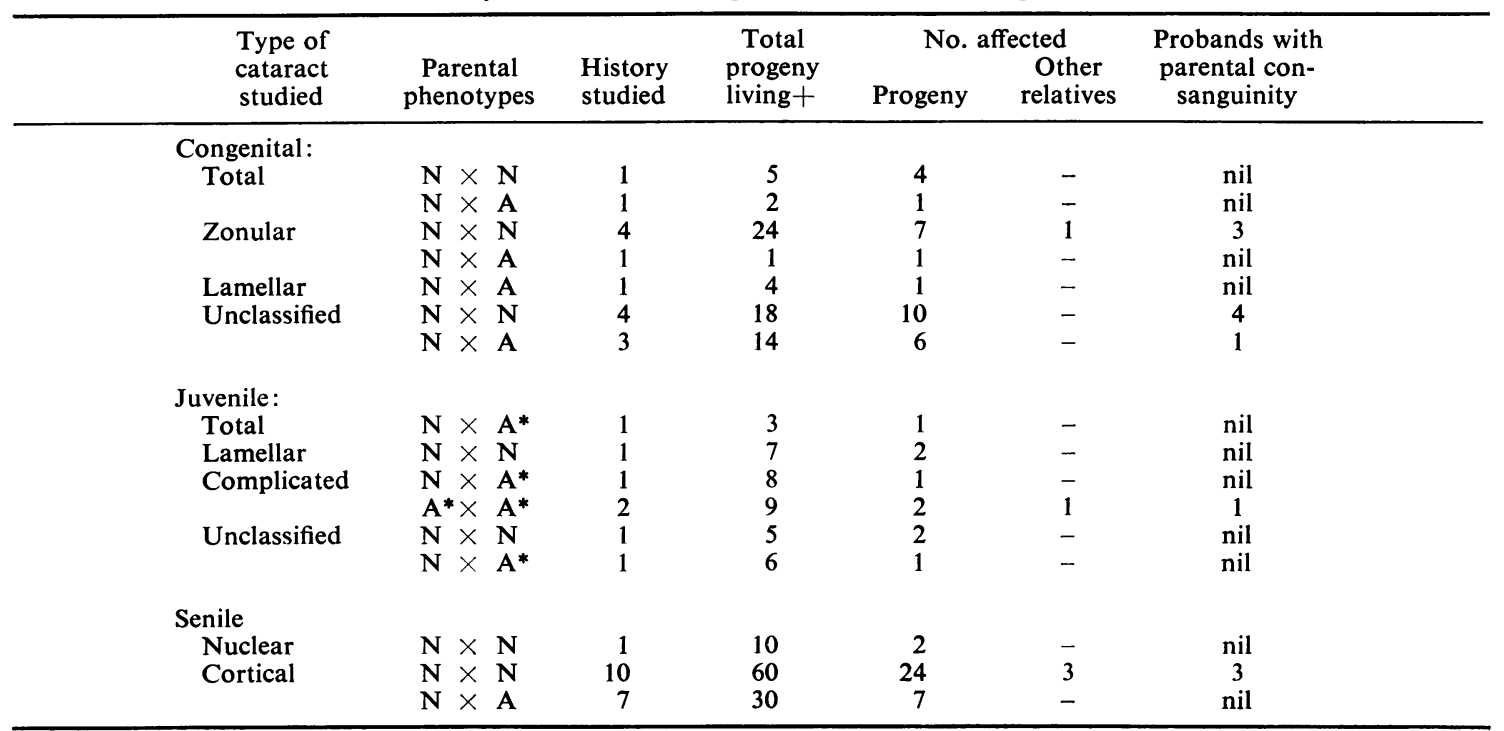


with low frequencies and those categorized as 'others' were not considered.

The patients and the controls showed no significant differences in their ethnic or caste distribution from the local population.

\section{Results}

$D$ line terminations. Cataracts in general and especially the cortical, congenital and senile types, showed significant differences from controls for D line terminations. There was a decrease in terminations ending at the 9th position in the 3 types, an increase in terminations at the 11 th position in the cortical and senile types, and a decrease in terminations ending at the 11 th position in the congenital cases (Table 3). These observations reflect heterogeneity between the 3 types of cataract in the D line terminations.

$C$ line terminations. Terminations of $\mathrm{C}$ line in zonular, cortical and senile cataracts differed significantly from the controls (Table 4) owing

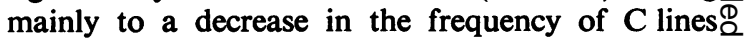
ending at the 7th position especially in the zonular. type. There was also a similar decrease in term- $\vec{F}$ inations ending at the 9th position in cortical and $\stackrel{\text { ? }}{9}$ senile cases. In general, $\mathrm{C}$ lines ended more? frequently in all cataracts at positions other than $\frac{\bar{O}}{\frac{0}{0}}$ the 5th, 7th and 9th.

$B$ line terminations. Terminations of $\mathrm{B}$ lines in nuclear, cortical and senile cataracts differed ${ }^{\infty}$ significantly from those in controls. This was mainly $\vec{O}$ due to a decrease of the terminations ending at the $5^{\prime}$ position in the 3 cataract types and an increase in $\omega_{\sigma}$ the terminations ending at the 7th position in nuclear and at the 9th position in cortical and senile 3 types compared with controls (Table 5).

A line terminations. Terminations of A lines showed variations in all cataracts from controls (Table 6). The frequency of terminations ending at the $3 \mathrm{rd}$ \%

TABLE 3. Distribution of $D$ line terminations in patients with cataract

\begin{tabular}{|c|c|c|c|c|c|c|c|c|c|c|}
\hline & & & & Po & of term & ions & & & & \\
\hline & & & 7 & 9 & 11 & Other & Total & D v. C & F v. I & 움 \\
\hline Type of & aract: & & & & & & & & & \\
\hline Total & & $\begin{array}{l}\mathbf{N} \\
\%\end{array}$ & $\begin{array}{c}4 \\
22 \cdot 2\end{array}$ & $\begin{array}{c}5 \\
27 \cdot 8\end{array}$ & $\begin{array}{c}9 \\
50 \cdot 0\end{array}$ & $\begin{array}{l}0 \\
-\end{array}$ & 18 & $\begin{array}{c}1 \cdot 29 \\
(3)\end{array}$ & - & Oे \\
\hline Nuclear & & $\begin{array}{l}\mathrm{N} \\
\%\end{array}$ & $\begin{array}{c}3 \\
15 \cdot 0\end{array}$ & $\begin{array}{c}3 \\
15 \cdot 0\end{array}$ & $\begin{array}{l}13 \\
65 \cdot 0\end{array}$ & $\begin{array}{l}1 \\
5 \cdot 0\end{array}$ & 20 & $\begin{array}{c}5 \cdot 89 \\
(3)\end{array}$ & $-\leqq$ & סे \\
\hline Zonular & & $\begin{array}{l}\mathrm{N} \\
\%\end{array}$ & $\begin{array}{c}6 \\
21 \cdot 4\end{array}$ & $\begin{array}{c}9 \\
32 \cdot 1\end{array}$ & $\begin{array}{l}13 \\
46 \cdot 4\end{array}$ & $\begin{array}{l}0 \\
-\end{array}$ & 28 & $\begin{array}{c}1 \cdot 33 \\
(3)\end{array}$ & - & $\stackrel{+}{+}$ \\
\hline Cortical & & $\begin{array}{l}\mathrm{N} \\
\%\end{array}$ & $\begin{array}{l}41 \\
22 \cdot 5\end{array}$ & $\begin{array}{l}32 \\
17 \cdot 6\end{array}$ & $\begin{array}{l}93 \\
51 \cdot 1\end{array}$ & $\begin{array}{c}16 \\
8 \cdot 8\end{array}$ & 182 & $\begin{array}{c}22 \cdot 43^{* *} \\
\text { (3) }\end{array}$ & - & 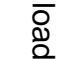 \\
\hline Complica & & $\begin{array}{l}\mathrm{N} \\
\%\end{array}$ & $\begin{array}{l}16 \\
38 \cdot 1\end{array}$ & $\begin{array}{l}13 \\
30 \cdot 9\end{array}$ & $\begin{array}{l}13 \\
30 \cdot 9\end{array}$ & $\begin{array}{l}0 \\
-\end{array}$ & 42 & $\begin{array}{c}6.03 \\
(3)\end{array}$ & - & $\frac{1}{0}$ \\
\hline Congenit & (F) & $\begin{array}{l}\mathrm{N} \\
\%\end{array}$ & $\begin{array}{l}12 \\
42 \cdot 9\end{array}$ & $\begin{array}{c}8 \\
28 \cdot 6\end{array}$ & $\begin{array}{c}7 \\
25 \cdot 0\end{array}$ & $\begin{array}{l}1 \\
3 \cdot 6\end{array}$ & 28 & - & - & $\begin{array}{l}\overrightarrow{\overline{0}} \\
\mathrm{3}\end{array}$ \\
\hline & (I) & $\begin{array}{l}\mathbf{N} \\
\%\end{array}$ & $\begin{array}{l}34 \\
32 \cdot 1\end{array}$ & $\begin{array}{l}26 \\
24 \cdot 5\end{array}$ & $\begin{array}{l}43 \\
40 \cdot 6\end{array}$ & $\begin{array}{l}3 \\
2 \cdot 8\end{array}$ & 106 & - & - & 晜 \\
\hline & $(F+I)$ & $\begin{array}{l}\mathbf{N} \\
\%\end{array}$ & $\begin{array}{l}46 \\
34 \cdot 3\end{array}$ & $\begin{array}{l}34 \\
25 \cdot 4\end{array}$ & $\begin{array}{l}50 \\
37 \cdot 3\end{array}$ & $\begin{array}{l}4 \\
2 \cdot 9\end{array}$ & 134 & $\begin{array}{l}7 \cdot 83^{*} \\
(3)\end{array}$ & $\begin{array}{c}2 \cdot 42 \\
(2)\end{array}$ & \\
\hline Juvenile & (F) & $\begin{array}{l}\mathrm{N} \\
\%\end{array}$ & $\begin{array}{c}7 \\
50 \cdot 0\end{array}$ & $\begin{array}{c}3 \\
21 \cdot 4\end{array}$ & $\begin{array}{c}4 \\
28 \cdot 6\end{array}$ & $\begin{array}{l}0 \\
-\end{array}$ & 14 & - & - & e. \\
\hline & (I) & $\begin{array}{l}\mathrm{N} \\
\%\end{array}$ & $\begin{array}{l}12 \\
26 \cdot 1\end{array}$ & $\begin{array}{l}16 \\
34 \cdot 8\end{array}$ & $\begin{array}{l}18 \\
39 \cdot 1\end{array}$ & $\begin{array}{l}0 \\
-\end{array}$ & 46 & - & - & \\
\hline & $(F+I)$ & $\begin{array}{l}\mathrm{N} \\
\%\end{array}$ & $\begin{array}{l}19 \\
31 \cdot 7\end{array}$ & $\begin{array}{l}19 \\
31 \cdot 7\end{array}$ & $\begin{array}{l}22 \\
36 \cdot 7\end{array}$ & $\begin{array}{l}0 \\
-\end{array}$ & 60 & $\begin{array}{c}4 \cdot 29 \\
(3)\end{array}$ & $\begin{array}{c}2 \cdot 74 \\
(2)\end{array}$ & \\
\hline Senile & (F) & $\begin{array}{l}\mathrm{N} \\
\%\end{array}$ & $\begin{array}{c}5 \\
16 \cdot 7\end{array}$ & $\begin{array}{l}2 \\
6 \cdot 7\end{array}$ & $\begin{array}{l}22 \\
73 \cdot 3\end{array}$ & $\begin{array}{l}1 \\
3 \cdot 3\end{array}$ & 30 & - & - & \\
\hline & (I) & $\begin{array}{l}\mathbf{N} \\
\%\end{array}$ & $\begin{array}{l}47 \\
25 \cdot 0\end{array}$ & $\begin{array}{l}37 \\
19 \cdot 7\end{array}$ & $\begin{array}{l}89 \\
47 \cdot 3\end{array}$ & $\begin{array}{l}15 \\
7 \cdot 9\end{array}$ & 188 & - & - & \\
\hline & $(F+I)$ & $\begin{array}{l}\mathrm{N} \\
\%\end{array}$ & $\begin{array}{l}52 \\
23.8\end{array}$ & $\begin{array}{l}39 \\
17 \cdot 9\end{array}$ & $\begin{array}{l}1.11 \\
50.9\end{array}$ & $\begin{array}{l}16 \\
7 \cdot 3\end{array}$ & 218 & $\begin{array}{c}21 \cdot 25^{* *} \\
\text { (3) }\end{array}$ & $\begin{array}{l}6.97 * * \\
(2)\end{array}$ & * \\
\hline Total & (F) & $\begin{array}{l}\mathrm{N} \\
\%\end{array}$ & $\begin{array}{l}24 \\
33 \cdot 3\end{array}$ & $\begin{array}{l}13 \\
18 \cdot 1\end{array}$ & $\begin{array}{l}33 \\
45 \cdot 8\end{array}$ & $\begin{array}{l}2 \\
2 \cdot 8\end{array}$ & 72 & - & - & \\
\hline & (I) & $\begin{array}{l}\mathbf{N} \\
\%\end{array}$ & $\begin{array}{l}93 \\
27 \cdot 4\end{array}$ & $\begin{array}{l}79 \\
23 \cdot 2\end{array}$ & $\begin{array}{l}150 \\
44 \cdot 1\end{array}$ & $\begin{array}{c}18 \\
5 \cdot 3\end{array}$ & 340 & - & - & 0 \\
\hline & $(F+I)$ & $\begin{array}{l}\mathbf{0} \\
\%\end{array}$ & $\begin{array}{c}117 \\
28 \cdot 4\end{array}$ & $\begin{array}{l}92 \\
22 \cdot 3\end{array}$ & $\begin{array}{c}183 \\
44 \cdot 4\end{array}$ & $\begin{array}{c}20 \\
4.9\end{array}$ & 412 & $\begin{array}{c}13 \cdot 96^{*} \\
\text { (3) }\end{array}$ & $\begin{array}{c}1.49 \\
(2)\end{array}$ & $\stackrel{0}{\frac{0}{\sigma}}$ \\
\hline Control & & $\begin{array}{l}\mathrm{N} \\
\%\end{array}$ & $\begin{array}{l}43 \\
21 \cdot 7\end{array}$ & $\begin{array}{l}70 \\
35 \cdot 4\end{array}$ & $\begin{array}{l}81 \\
40.9\end{array}$ & $\begin{array}{l}4 \\
2 \cdot 9\end{array}$ & 198 & - & - & \\
\hline
\end{tabular}

$\mathrm{N}=$ number of palms studied, $\mathrm{F}=$ familial, $\mathrm{I}=$ isolated, $\mathrm{D}=$ disease, $\mathrm{C}=$ control, $* P<0.05, * * P<0.01$. Figures in parenthesis $\mathrm{O}$ are degrees of freedom. 
TABLE 4. Distribution of $C$ line terminations in patients with cataract

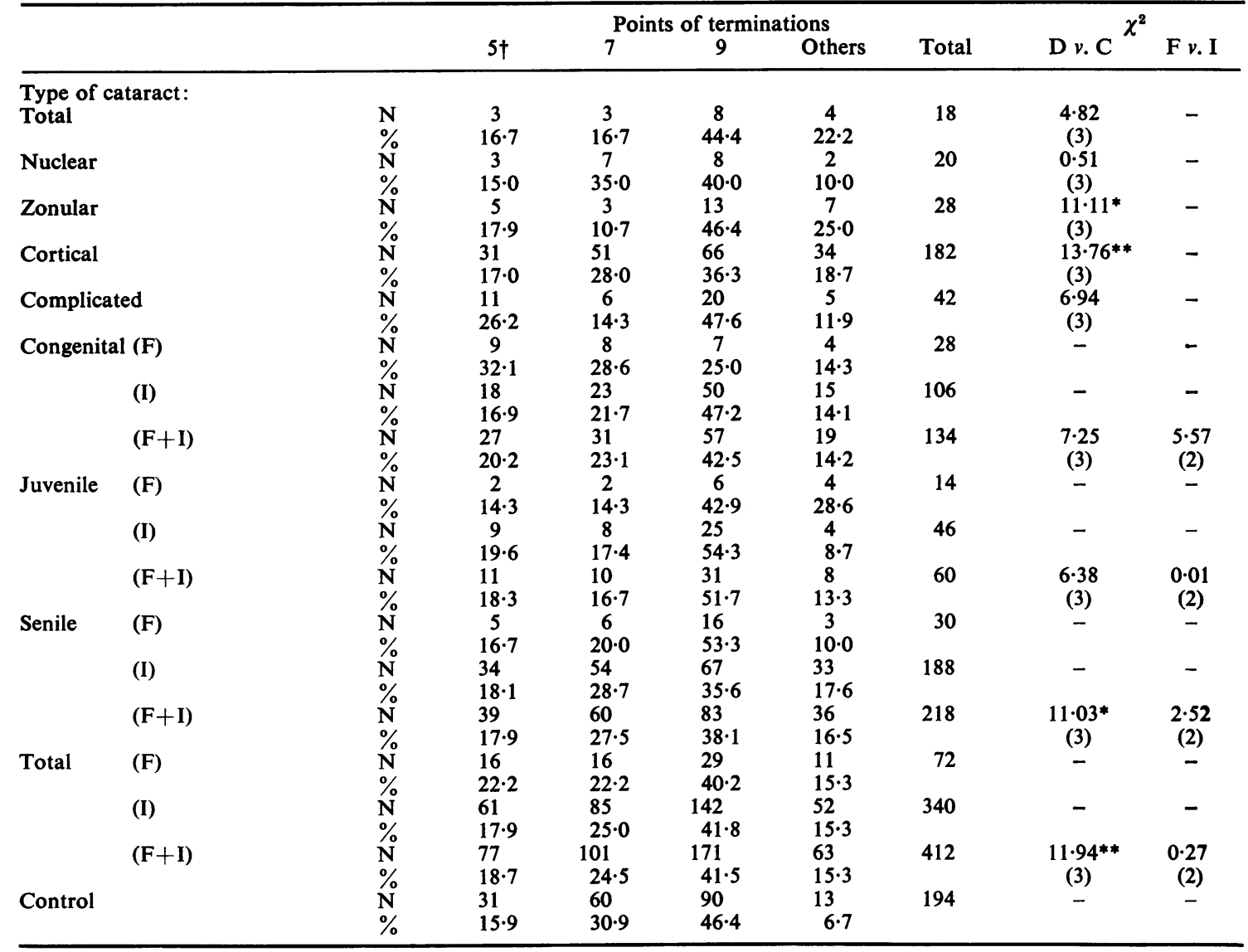

$\mathrm{N}=$ number of palms studied, $\mathrm{F}=$ familial, $\mathrm{I}=$ isolated, $\mathrm{D}=$ disease, $\mathrm{C}=$ control. $* P<0.05, * * P<0.01$. Figures in parenthesis are degrees of freedom. †Includes terminations ending at $5^{\prime}$ and $5^{\prime \prime}$ positions.

position were strikingly less with a corresponding increase in the terminations ending at the 4th, $5^{\prime}$ and $5^{\prime \prime}$ positions.

Main line terminations in familial and isolated cases. A comparison of familial and isolated cases showed significant differences in the terminations of $D$ and $A$ lines in senile cataracts alone (Tables 3 to 6). In congenital and juvenile cataracts, such differences were less striking; those found in senile cases were mainly due to a decrease in the terminations of $D$ line endings at the 7th and 9th positions with a corresponding increase of terminations at the 11th position in the familial group compared with the isolated group ( $\chi^{2} 6.972$ d.f., $\left.P<0.01\right)$. Regarding the $\mathbf{A}$ line, there was a reduction in the terminations at the 3rd and $5^{\prime}$ position and an increase at the 4th and $5^{\prime \prime}$ positions in familial senile cataracts compared with isolated senile cataracts $\left(\chi^{2} \quad 9.42\right.$ 3 d.f., $P<0.05)$.

\section{Distribution of main line terminations according to} sex. Some nonspecific variations between the sexes were observed in the terminations of main lines. The control group showed significant differences in the terminations of $\mathrm{B}$ lines ending at $5^{\prime}$ position (higher in females) and $5^{\prime \prime}$ positions (higher in males) and in the terminations of $A$ lines ending at almost all positions (Tables 9 and 10).

Significant sex differences were found in the congenital group (C line terminations, $\chi^{2} 12.53$ 3 d.f., $P<0.05)$ and in the juvenile group (C line termination $\chi^{2} 9.613$ d.f., $P<0.05$; A line termination $\chi^{2} 10.583$ d.f., $\left.P<0.05\right)$. Sex variations were not observed in cases of senile cataract.

Comparison of line terminations for each sex in 
TABLE 5. Distribution of B line terminations in patients with cataract

\begin{tabular}{|c|c|c|c|c|c|c|c|c|c|c|c|}
\hline & & & & & oints of & inatior & & & $\chi$ & & \\
\hline & & & $5^{\prime}$ & $5^{\prime \prime}$ & 7 & 9 & Others & Total & $\mathrm{D} v \cdot \mathrm{C}^{\lambda}$ & F v. I & 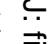 \\
\hline Type of $c$ & taract: & & & & & & & & & & \\
\hline Total & & $\mathrm{N}$ & 2 & 5 & 10 & 0 & 1 & 18 & 5.91 & - & Oֵ \\
\hline & & $\%$ & $11 \cdot 1$ & $27 \cdot 8$ & $55 \cdot 6$ & - & $5 \cdot 6$ & & (4) & & o \\
\hline Nuclear & & $\mathrm{N}$ & 2 & 4 & 12 & 0 & 2 & 20 & $18 \cdot 69 * *$ & - & $\overline{\overline{\bar{\phi}}}$ \\
\hline & & $\%$ & 10.0 & $20 \cdot 0$ & $60 \cdot 0$ & - & $10 \cdot 0$ & & (4) & & $\vec{\nabla}$ \\
\hline Zonular & & $\mathrm{N}$ & 3 & 8 & 16 & 0 & 1 & 28 & $6 \cdot 60$ & - & a \\
\hline & & $\%$ & $10 \cdot 7$ & $28 \cdot 6$ & $57 \cdot 1$ & - & $3 \cdot 6$ & & (4) & & ڤు \\
\hline Cortical & & $\begin{array}{l}\mathrm{N} \\
\%\end{array}$ & $\begin{array}{l}40 \\
22 \cdot 0\end{array}$ & $\begin{array}{l}38 \\
20 \cdot 9\end{array}$ & $\begin{array}{l}77 \\
42 \cdot 3\end{array}$ & $\begin{array}{l}15 \\
8 \cdot 2\end{array}$ & $\begin{array}{l}12 \\
6 \cdot 6\end{array}$ & 182 & $\begin{array}{c}11 \cdot 03^{*} \\
(4)\end{array}$ & - & 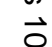 \\
\hline Complica & & $\mathbf{N}$ & 10 & 15 & 14 & 2 & 1 & 42 & $5 \cdot 87$ & - & \\
\hline & & $\%$ & $23 \cdot 8$ & $35 \cdot 7$ & $33 \cdot 3$ & $4 \cdot 8$ & $2 \cdot 4$ & & (4) & & \\
\hline Congenit & $(F)$ & $\mathbf{N}$ & 8 & 10 & 7 & 0 & 3 & 28 & - & - & 0 \\
\hline & & $\%$ & $28 \cdot 7$ & $35 \cdot 7$ & $25 \cdot 0$ & - & $10 \cdot 7$ & & & & 8 \\
\hline & (I) & $\mathbf{N}$ & 23 & 32 & 46 & 0 & 5 & 106 & - & - & 3 \\
\hline & & $\%$ & $21 \cdot 7$ & $30 \cdot 1$ & $43 \cdot 4$ & - & $4 \cdot 7$ & & & & \\
\hline & $(F+I)$ & $\mathbf{N}$ & 31 & 42 & 53 & 0 & 8 & 134 & $5 \cdot 54$ & $2 \cdot 66$ & \\
\hline & & $\%$ & $23 \cdot 1$ & $31 \cdot 3$ & $39 \cdot 6$ & - & $6 \cdot 0$ & & (3) & (2) & ') \\
\hline Juvenile & (F) & $\begin{array}{l}\mathrm{N} \\
\%\end{array}$ & $\begin{array}{c}3 \\
21 \cdot 4\end{array}$ & $\begin{array}{c}3 \\
21 \cdot 4\end{array}$ & $\begin{array}{c}6 \\
42 \cdot 9\end{array}$ & $\begin{array}{c}2 \\
14 \cdot 3\end{array}$ & $\begin{array}{l}0 \\
-\end{array}$ & 14 & - & - & প \\
\hline & (1) & $\mathbf{N}$ & 11 & 16 & 16 & 0 & 3 & 46 & - & - & م \\
\hline & & $\%$ & $23 \cdot 9$ & $34 \cdot 8$ & $34 \cdot 8$ & - & $6 \cdot 5$ & & & & \\
\hline & $(F+I)$ & $\mathbf{N}$ & 14 & 19 & 22 & 2 & 3 & 60 & $4 \cdot 08$ & 0.79 & כ \\
\hline & & $\%$ & $23 \cdot 3$ & $31 \cdot 7$ & $36 \cdot 7$ & $3 \cdot 3$ & $5 \cdot 0$ & & (3) & (2) & \\
\hline Senile & (F) & $\begin{array}{l}\mathrm{N} \\
0\end{array}$ & $\begin{array}{c}5 \\
16.7\end{array}$ & 3 & 20 & $\begin{array}{l}2 \\
6.7\end{array}$ & 0 & 30 & - & - & \\
\hline & (I) & $\mathbf{N}$ & $\begin{array}{l}16.1 \\
37\end{array}$ & $\begin{array}{l}10 \cdot 0 \\
47\end{array}$ & $\begin{array}{l}66 \cdot 7 \\
73\end{array}$ & 14 & $1 \overline{7}$ & 188 & - & - & \\
\hline & & $\%$ & $19 \cdot 7$ & 25.0 & $38 \cdot 8$ & $7 \cdot 5$ & $9 \cdot 0$ & & & & U \\
\hline & $(F+I)$ & $\mathbf{N}$ & 42 & 50 & 93 & 16 & 17 & 218 & $13 \cdot 05^{* *}$ & $7 \cdot 160$ & \\
\hline & & $\%$ & $19 \cdot 3$ & $22 \cdot 9$ & $42 \cdot 7$ & $7 \cdot 3$ & $7 \cdot 8$ & & (3) & (3) $\frac{0}{0}$ & \\
\hline Total & & $\mathrm{N}$ & $\begin{array}{l}16 \\
22 \cdot 2\end{array}$ & $\begin{array}{l}16 \\
22 \cdot 2\end{array}$ & $\begin{array}{l}33 \\
45 \cdot 8\end{array}$ & $\begin{array}{l}4 \\
5 \cdot 6\end{array}$ & $\begin{array}{l}3 \\
4 \cdot 2\end{array}$ & 72 & - & $-\grave{\bar{a}}$ & \\
\hline & (I) & $\mathbf{N}_{0}^{\mathbf{o}}$ & 71 & 95 & 135 & 14 & 25 & 340 & - & $-\stackrel{2}{?}$ & +0 \\
\hline & $(\mathrm{F}, \mathrm{J}$ & $\%$ & $20 \cdot 9$ & $27 \cdot 9$ & $39 \cdot 7$ & $4 \cdot 1$ & $7 \cdot 4$ & & & & \\
\hline & $(F+1)$ & $\begin{array}{l}\mathrm{N} \\
\%\end{array}$ & $\begin{array}{l}27 \\
21 \cdot 1\end{array}$ & $\begin{array}{l}111 \\
26 \cdot 9\end{array}$ & $\begin{array}{l}168 \\
40 \cdot 8\end{array}$ & $\begin{array}{l}18 \\
4 \cdot 4\end{array}$ & $\begin{array}{l}28 \\
6 \cdot 8\end{array}$ & 412 & $\begin{array}{c}10 \cdot 56^{*} \\
(3)\end{array}$ & $\begin{array}{c}1 \cdot 36 \\
(2)\end{array}$ & עִ \\
\hline Control & & $\begin{array}{l}\mathrm{N} \\
\%\end{array}$ & $\begin{array}{l}58 \\
29 \cdot 3\end{array}$ & $\begin{array}{l}40 \\
20 \cdot 2\end{array}$ & $\begin{array}{l}88 \\
44 \cdot 4\end{array}$ & $\begin{array}{l}4 \\
2 \cdot 0\end{array}$ & $\begin{array}{l}8 \\
4 \cdot 0\end{array}$ & 198 & - & - & \\
\hline
\end{tabular}

$\mathrm{N}=$ number of palms studied, $\mathrm{F}=$ familial, $\mathrm{I}=$ isolated, $\mathrm{D}=$ disease, $\mathrm{C}=$ control. ${ }^{*} P<0.05,{ }^{* *} P<0 \cdot 01$. Figures in parenthesis are degrees of freedom.

the disease sub-groups with the corresponding controls showed significant differences for (a) male congenital cataract (C line, $\chi^{2} 8.743$ d.f., $P<0.05$; A line, $\chi^{2} 11.463$ d.f., $P<0.05$ ); (b) male juvenile cataract (A line, $\chi^{2} 13.314$ d.f., $P<0.01$ ); (c) male senile only (B line $\chi^{2} 9.994$ d.f., $P<0.05$ ).

Female congenital and senile cases differed significantly from controls in the terminations of all 4 main lines while juvenile cataracts showed the difference only for B and A lines (Tables 7 to 10).

These findings indicate a possible sex-dependent heterogeneity in the main line terminations in cataracts.

\section{Discussion}

A comparative analysis of the alignment of 4 main lines (DCBA) in the present study showed that senile and cortical types differ from the controls in terminations of all 4. This similarity between senile and cortical types must be due to the high proportion $(80 \%)$ of cortical types occurring in the senile cases. Among other cataracts compared with controls, congenital types differed only in the $D$ and $\mathrm{A}$ line terminations while zonular types differed $\mathrm{B}$. in $\mathbf{C}$ and $\mathrm{A}$ lines, and nuclear types in $\mathrm{B}$ and $\mathrm{A}$ line terminations. All other types of cataract differed only in A line terminations. This indicates that theo association of main line terminations is specific to the type of cataract, and probably is influenced by을 the pleiotropic mechanism of the genes and the other factors controlling the type of cataract.o It also suggests that there is an effect of a monogenic N condition such as cataract on a polygenic character ${ }_{\mathcal{E}}$ such as dermatoglyphics. The association betweeno main line polymorphisms and ocular defects does not appear to have been reported previously.

The differences found between the familial and $\stackrel{\oplus}{\rightarrow}$ isolated cases suggest the possibility of considering 0 $\mathbf{D}$ and $\mathbf{A}$ line terminations in the identification of $\bar{O}$ familial senile cataracts. 
TABLE 6. Distribution of $\mathrm{A}$ line terminations in patients with cataract

\begin{tabular}{|c|c|c|c|c|c|c|c|c|c|c|}
\hline & & & & & Points o & inatio & & & & \\
\hline & & & 3 & 4 & $5^{\prime}$ & $5^{\prime \prime}$ & Others & Total & $\mathrm{D} v \mathrm{C}$ & F v. I \\
\hline Type of & taract: & & & & & & & & & \\
\hline Total & & $\mathrm{N}$ & 3 & 4 & 4 & 3 & 4 & 18 & $19 \cdot 21^{*}$ & - \\
\hline Nuclear & & $N$ & $\begin{array}{c}16 \cdot 7 \\
5\end{array}$ & $\begin{array}{c}22 \cdot 2 \\
3\end{array}$ & $\begin{array}{c}22 \cdot 2 \\
6\end{array}$ & $\begin{array}{c}16 \cdot 7 \\
2\end{array}$ & $\begin{array}{c}22 \cdot 2 \\
4\end{array}$ & & (4) & \\
\hline & & $\%$ & $25 \cdot 0$ & $15 \cdot 0$ & $30 \cdot 0$ & $10 \cdot 0$ & $20 \cdot 0$ & 20 & $\begin{array}{c}12 \cdot 60^{* *} \\
(4)\end{array}$ & - \\
\hline Zonular & & $\mathrm{N}$ & 10 & 5 & 9 & 0 & 4 & 28 & $39 \cdot 06^{* *}$ & - \\
\hline & & $\%$ & $35 \cdot 7$ & $17 \cdot 9$ & $32 \cdot 1$ & - & $14 \cdot 3$ & & (4) & \\
\hline Cortical & & $\mathrm{N}$ & 76 & 42 & 48 & 8 & 8 & 182 & $22 \cdot 61 * *$ & \\
\hline & & $\%$ & $41 \cdot 8$ & $23 \cdot 1$ & $26 \cdot 4$ & $4 \cdot 4$ & $4 \cdot 4$ & & (4) & \\
\hline Complica & & $\mathrm{N}$ & 13 & 12 & 11 & 2 & 4 & 42 & $16 \cdot 56^{* *}$ & - \\
\hline & & $\%$ & $31 \cdot 0$ & $28 \cdot 6$ & $26 \cdot 2$ & $4 \cdot 8$ & $9 \cdot 5$ & & (4) & \\
\hline Congenit: & (F) & $\begin{array}{l}\mathrm{N} \\
0\end{array}$ & 11 & 4 & 10 & 0 & 3 & 28 & - & - \\
\hline & (I) & $\stackrel{0}{N}$ & 34 & $\begin{array}{l}14 \cdot 3 \\
19\end{array}$ & $\begin{array}{l}35 \cdot 7 \\
34\end{array}$ & $\overline{0}$ & $\begin{array}{l}10 \cdot 7 \\
19\end{array}$ & 106 & - & - \\
\hline & & $\%$ & $32 \cdot 1$ & $17 \cdot 9$ & $32 \cdot 1$ & - & $17 \cdot 9$ & & & \\
\hline & $(\mathrm{F} \mapsto \mathrm{I})$ & $\mathrm{N}$ & 45 & 23 & 44 & 0 & 22 & 134 & $30 \cdot 06^{* *}$ & 0.46 \\
\hline & & $\%$ & $33 \cdot 6$ & $17 \cdot 2$ & $32 \cdot 8$ & - & $16 \cdot 4$ & & (3) & (2) \\
\hline Juvenile & $(F)$ & $\mathrm{N}$ & 3 & 5 & 6 & 0 & 0 & 14 & - & - \\
\hline & & $\%$ & $21 \cdot 4$ & $35 \cdot 7$ & $42 \cdot 9$ & - & - & & & \\
\hline & (I) & $\mathrm{N}$ & 10 & 19 & 8 & 0 & 9 & 46 & -. & - \\
\hline & & $\%$ & $21 \cdot 7$ & $41 \cdot 3$ & $17 \cdot 4$ & - & $19 \cdot 6$ & & & \\
\hline & $(F+1)$ & $\mathrm{N}$ & 13 & 24 & 14 & 0 & 9 & 60 & $41 \cdot 98^{* *}$ & $2 \cdot 21$ \\
\hline & & $\%$ & $21 \cdot 7$ & $40 \cdot 0$ & $23 \cdot 3$ & - & $15 \cdot 0$ & & (3) & (2) \\
\hline Senile & (F) & $\begin{array}{l}\mathrm{N} \\
0\end{array}$ & $\begin{array}{l}10 \\
33 \cdot 3\end{array}$ & 8 & 6 & 5 & 1 & 30 & - & - \\
\hline & (I) & $\mathrm{N}$ & $\begin{array}{l}33 \cdot 3 \\
81\end{array}$ & $\begin{array}{l}26 \cdot 7 \\
38\end{array}$ & $20 \cdot 0$ & $16 \cdot 7$ & $3 \cdot 3$ & & & \\
\hline & & $\%$ & $43 \cdot 1$ & $\begin{array}{l}38 \\
20 \cdot 2\end{array}$ & $\begin{array}{l}55 \\
29 \cdot 3\end{array}$ & $\begin{array}{l}5 \\
2 \cdot 7\end{array}$ & $\begin{array}{l}9 \\
4 \cdot 8\end{array}$ & 188 & - & - \\
\hline & $(F+I)$ & N & 91 & 46 & 61 & 10 & 10 & 218 & $23 \cdot 25 * *$ & $9 \cdot 42^{*}$ \\
\hline & & $\%$ & $41 \cdot 7$ & $21 \cdot 1$ & $28 \cdot 0$ & $4 \cdot 6$ & $4 \cdot 6$ & & (3) & (3) \\
\hline Total & $(F)$ & $\mathrm{N}$ & 24 & 17 & 22 & 5 & 4 & 72 & - & - \\
\hline & & $\%$ & $33 \cdot 3$ & $23 \cdot 6$ & $30 \cdot 6$ & $6 \cdot 9$ & $5 \cdot 6$ & & & \\
\hline & (I) & $\mathbf{N}$ & 125 & 76 & 97 & 5 & 37 & 340 & -- & - \\
\hline & & $\%$ & $36 \cdot 8$ & $22 \cdot 4$ & $28 \cdot 5$ & $1 \cdot 5$ & $10 \cdot 9$ & & & \\
\hline & $(F+I)$ & $\mathrm{N}_{0}^{\mathrm{N}}$ & 149 & 93 & 119 & 10 & 41 & 412 & $41 \cdot 18^{* *}$ & $5 \cdot 56$ \\
\hline & & $\%$ & $36 \cdot 2$ & $22 \cdot 6$ & $28 \cdot 9$ & $2 \cdot 4$ & $10 \cdot 0$ & & (3) & (3) \\
\hline Control & & $\mathrm{N}$ & 125 & 20 & 36 & 5 & 12 & 198 & $\cdots$ & \\
\hline & & $\%$ & $63 \cdot 1$ & $10 \cdot 1$ & $18 \cdot 1$ & $2 \cdot 5$ & $6 \cdot 1$ & & & \\
\hline
\end{tabular}

$\mathrm{N}$ - number of palms studied, $\mathrm{F}=$ familial, $\mathrm{I}-$ isolated, $\mathrm{D}$ disease, $\mathrm{C}=$ control. ${ }^{*} P<0.05, * * P-0.01$. Figures in parenthesis are degrees of freedom.

It is difficult at this time to offer any explanation for the male to female heterogeneity observed in the present study as no information is available on the main line polymorphisms in the 2 sexes either in the normal population (especially from the local population) or those suffering from other diseases. Sex variations are however reported for other dermatoglyphic characters such as finger and palmar patterns (Holt, 1968; Schauman and Alter, 1976).

The present findings indicate the necessity for further investigations on the main line polymorphisms in common diseases.

\section{Acknowledgments}

We thank Professor O. S. Reddi, former Head, Department of Genetics, Osmania University, and Dr P. Siva Reddy, Director, Sarojini Devi Eye Hospital and Institute of Ophthalmology, Hyderabad, for providing the necessary facilities. Our thanks are also due to the ophthalmologists concerned in diagnosing the cases included in the study.

\section{References}

Cummins, H. \& Midlo, C. (1943) Finger Prints, Palms and Soles. The Blackiston Company, Philadelphia.

Eswariah, G. \& BALI, R.S. (1977) Palmar flexion creases and dermatoglyphics among diabetic patients. American Journal of Physical Anthropology, 47, 11.

François, J., Matton-van Leuven, M.T. \& Debie, S. (1969) Dermatoglyphics in retinoblastoma. Journal de génétique humaine, 17, 367.

Holt, S.B. (1968) The Genetics of Dermal Ridges. Charles Thomas, Illinois, USA.

MURTY, J.S. (1973) Genetic factors in senile cataracts. Eastern Archives of Ophthalmology, 1, 1.

Padma, T., Murty, J.S., Lakshmamma, K. \& Siva Reddy, P. (1977) Patterns of inheritance in cataract. Eastern Archives of Ophthalmology, 4, 59.

Schauman, B. \& Alter, M. (1976) Dermatoglyphics in Medical Disorders. Springer-Verlag, New York.

Vidal, O.R., Damel, A. \& Funes, J.C. (1969) Dermatoglyphics in retinoblastoma. Journal de génétique humaine, $17,19$. 


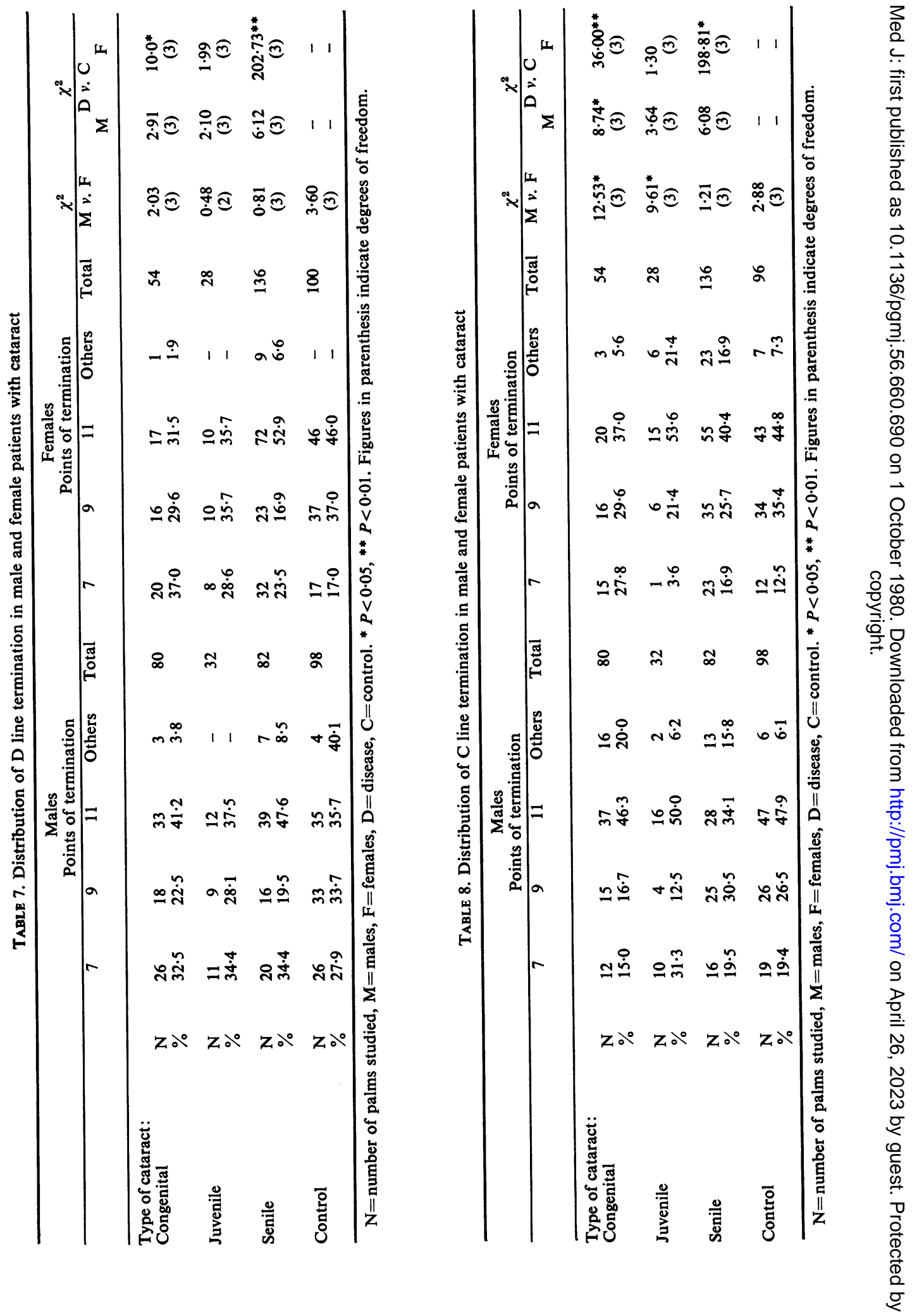



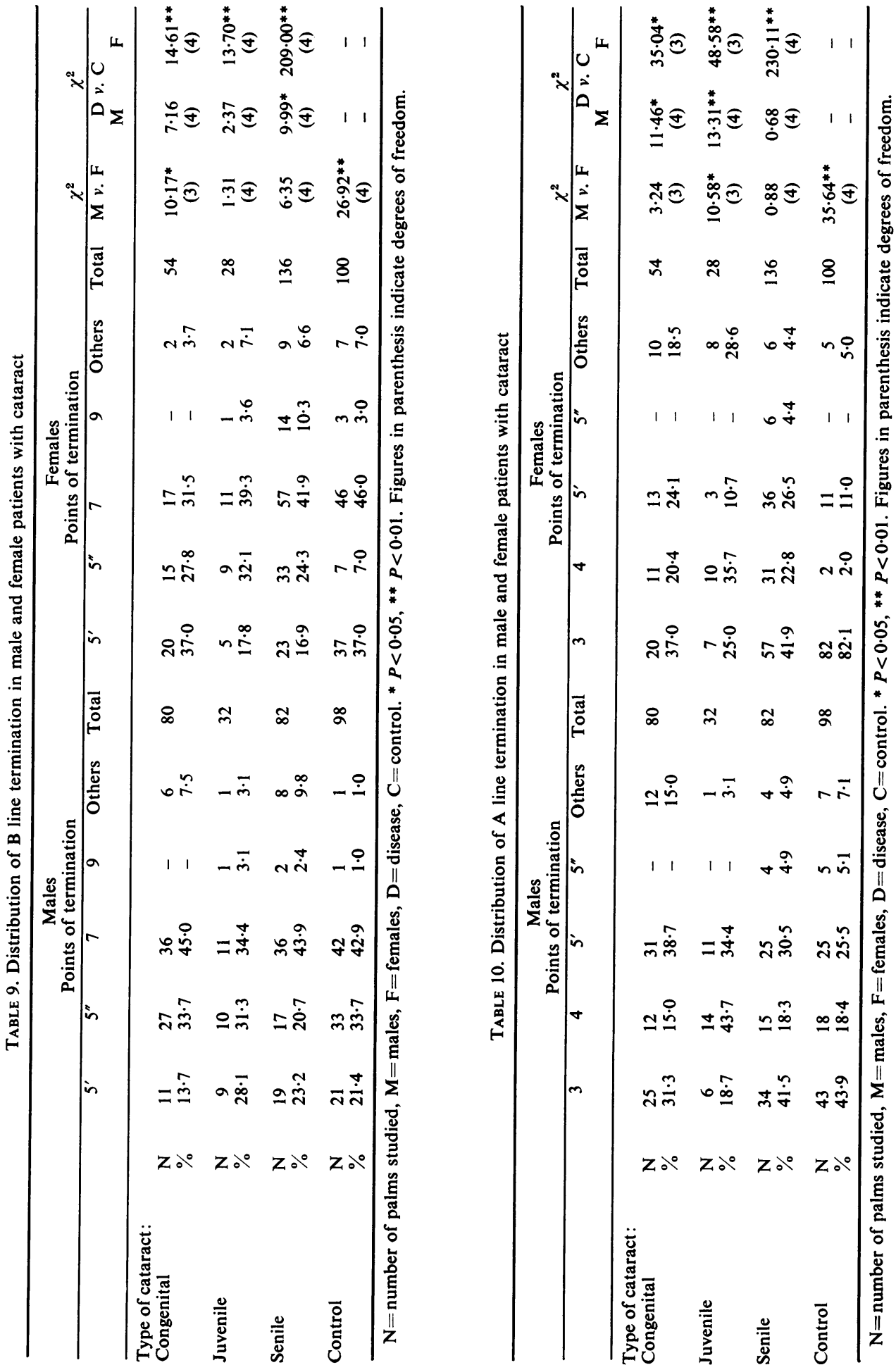